\title{
The Buffer Effect of Receiving Social Support on SNS Exhaustion and SNS Satisfaction: An Exploratory Study of the Lonely and Emotionally Unstable
}

\author{
Janice Lo \\ Creighton University \\ janicelo@creighton.edu
}

\author{
Chengqi Guo \\ James Madison University \\ guocx@jmu.edu
}

\author{
Bree Bradley \\ James Madison University \\ bradlebn@dukes.jmu.edu
}

\begin{abstract}
Popular press reports that using social networking sites (SNSs) can cause increased stress levels in individuals. Although academic literature has attempted to explain the technostress related to social media, much remains unclear how feelings of being drained from SNS activities can be alleviated. This exploratory study draws upon the buffer effect of social support theory and tests it in the context of SNS. We analyze users who experience loneliness and emotional instability to better understand their psychological responses to social overload and receiving social support in terms of effects on SNS satisfaction, exhaustion, and discontinuous usage intention. Data from more than 1,000 users support our hypotheses that receiving social support is significantly related to SNS exhaustion and satisfaction. Interestingly, contrary to prior studies, the relationship between social overload and SNS satisfaction is found to be significantly positive for emotionally unstable users. Implications for research and practice are provided.
\end{abstract}

\section{Introduction}

In recent years, the terms "technostress", "iDisorders", "digital detox", and "connection overload" have become prominent in the popular literature [e.g. 8, 19, 38, 41]. Many people express the need to rejuvenate themselves by removing Internet-connected devices for an interval [e.g. 41] and especially at the forefront of their "digital detox" plan is the need to disconnect or reduce their use of social media in particular [e.g. 8, 38]. This phenomenon of disconnecting or reducing the use of social media, particularly the use of social networking sites (SNSs), has penetrated the academic literature in recent years as well [e.g. 2, 26, 27]. Many users intend to disconnect due to the strain, fatigue, and other problems arising from social media use [26]. This fatigue, or SNS exhaustion, refers to users' feelings of being drained from SNS usage- related activities [26]. SNS exhaustion, along with the level of satisfaction users have with a SNS, have been found to be major determinants (positive and negative, respectively) of users' intentions to discontinue using or to reduce the usage of a SNS $[25,26]$. Studies have identified that one of the major antecedents to SNS exhaustion is social overload, the feeling of providing too much social support to others in one's social network out of a sense of duty $[25,26]$. While studies such as $[25,26$, 27] have focused on the stress-related exhaustion of social media use and its consequences on discontinuous use, fewer studies seem to have offered factors that might help assuage this SNS exhaustion. In this paper, we draw from the field of medicine the buffer effect of social support theory $[1,7,33]$. The theory postulates that people who receive social support are able to better cope in situations of illness or other physical ailment $[1,33]$. In a similar sense, we suggest that receiving social support on a SNS may be a way to help reduce users' SNS exhaustion and enhance satisfaction with the SNS, and thereby relaxing users' intention to disconnect. Hence, the first purpose of this paper is to propose and test this in the context of SNS discontinuous usage intention.

But in the context of SNS, the effect of receiving social support is likely to unfold differently for people who feel lonely or who are emotionally unstable (i.e. neurotic). Studies have found that these particular personal traits are associated with problematic use of SNS [e.g. 14, 22, 42]. Emotionally unstable and lonely people tend to use SNSs to avoid loneliness and seek social support [14, $42,46]$, although rather than finding what they seek after, the result is the opposite. The time they spend on SNSs may be undermining their well-being and increasing their loneliness [22]. However, whether the social support they receive is associated with reduced exhaustion and increased satisfaction with the SNS remains to be seen. Thus the second purpose of this paper is to explore these effects for people who are lonely or emotionally unstable as opposed to people who are not. 
It is important to investigate how factors such as social overload and social support affect SNS exhaustion suffered by individuals experiencing psychological issues (e.g., long term tendency to be in a negative emotional state). For one, multiple studies suggest the causal relationship between changes in human behavior brought by social media and psychiatric disorders [32]. The scope of affected individuals is wide, ranging from the younger generation [11] to female adopters [10]. Second, SNS use has been considered a remedy to negative feelings such as loneliness because lonely people seek comfort and support on SNSs [14]. But does it always work? This dual-purpose study requires a two-stage analysis: 1) analysis of the general respondents, and 2) analysis of respondents who experience emotional instability or loneliness.

\section{Background}

\subsection{SNS Exhaustion, Satisfaction, and Discontinuous Usage Intention}

Several recent studies, most notably by Maier and colleagues [25, 26, 27], have examined the quitting intentions of SNS users. It was found that this discontinuous usage intention is strongly associated with two primary factors: SNS exhaustion and SNS satisfaction. The exhaustion a user feels from using a SNS is found to increase his or her quitting intention, and the satisfaction the user receives from using the SNS is found to reduce quitting intention $[25,26]$. Due to space limitation, we focus on reviewing exhaustion rather than satisfaction in this background section because it has both a direct and indirect effect (via SNS satisfaction) on discontinuous usage intention. In terms of exhaustion, studies have found that social overload, the feeling of providing too much social support to others in one's social network out of a sense of duty, is a major stressor causing feelings of SNS exhaustion or social media fatigue $[26,49]$. Other factors causing stress include having to deal with too many SNS system features and being overloaded with information presented by the SNS $[35,49]$. In addition to information overload, other factors like privacy concerns have also been found to be positively associated with SNS-related stress or fatigue [2]. Another factor that causes stress is social comparisons, which happens when users compare themselves to other SNS users [3, 13]. This comparison can have a detrimental impact on users. For example, Chou and Edge [6] found that longerterm Facebook users perceived other users as being happier and having better lives than themselves, which makes the longer-term users feel unhappy about themselves. But it does not stop there. Other studies have found that when users look at profiles of other, more attractive Facebook users, it made them feel bad about their own bodies [16]. The result was similarly harmful when male users view profiles of other males whom they perceive as having more successful careers [16]. All these factors contribute to SNS-related exhaustion, fatigue, or stress, which ultimately lead to SNS users' intention to disconnect.

\subsection{Buffer Effect of Social Support Theory}

The buffer effect of social support theory $[1,7$, $33,43]$ emerged from the field of medicine. It postulates that people who receive social support are able to better cope in situations of illness or other physical ailment [1,7]. For instance, Nucholls et al. [33] found that pregnant women who experienced high stress levels, but had social support were less likely to develop complications during pregnancy than those who did not have social support. And in a study of older people suffering from arthritis, Penninx et al. [36] found that having a variety of social support (e.g. from family, friends, partner) favorably influenced psychological functioning and reduced depressive symptoms, regardless of the severity level of their arthritic condition. In sports medicine, Mitchell et al. [28] found that injured athletes who perceived receiving support had reduced feelings of restlessness, isolation, and feeling cheated. Finally, in a study of early-stage cervical cancer survivors in Taiwan, Li et al. [23] found that having strong social support, along with high selfesteem, were positively associated with study participants' quality of life.

Because these studies suggest that receiving social support would enable people to better cope with stressful situations, we draw from this theory for the context of this study. We suggest that receiving social support on a SNS can be a way for SNS users to cope with SNS exhaustion and also enhance SNS satisfaction.

\section{Research Model, Hypotheses, and Exploratory Question}

Figure 1 depicts the overall research model for this paper. We hypothesize and test the concept that receiving social support helps alleviate the exhaustion experienced by SNS users and enhances their satisfaction with the SNS in the context of social overload and discontinuous usage intention. 


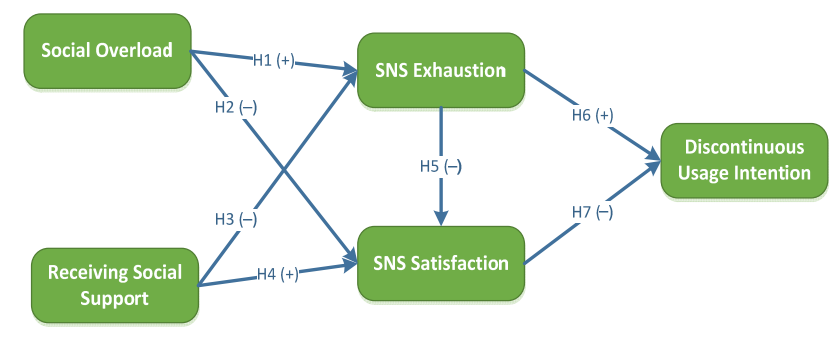

Figure 1. Research Model

\subsection{Associations between Social Overload and SNS Exhaustion and SNS Satisfaction}

SNS users experience social overload when they feel that they are providing too much social support to others in their social network, often out of a sense of duty $[25,26]$. The major purpose of an online social network is interaction among individuals of the network, which often includes people voicing their problems and other life situations to their network of online "friends", even if that situation is simply an upcoming birthday. This makes individuals in their network feel a sense of obligation to respond, even if the response is as simple as a small message acknowledging the birthday in terms of a happy birthday wish, for instance [13]. This sense of obligation to provide social support, however minor or major that support may be, creates the experience of social overload. Social overload is a psychological stressor, meaning that it induces feelings of fatigue. This fatigue transforms into feelings of SNS exhaustion, an unconscious and potentially harmful psychological reaction to stressful situations [26] resulting from the use of the SNS. Similar to the stressors that cause work exhaustion in employees in a work-related context, which ultimately leads to employee turnover intention [30], social overload is a stressor in the context of SNS use, leading to exhaustion from using the SNS. Therefore, we hypothesize:

\section{H1: Social overload is positively related to SNS exhaustion.}

Because social overload is seen as a psychological stressor, the consequences of social overload would be negative in terms of SNS satisfaction. So not only would social overload be expected to increase SNS exhaustion, it would also be expected to lead to dissatisfaction with using the SNS. Having too many requests for social support tends to become burdensome and therefore reduces users' sense of satisfaction with the SNS. It is thus hypothesized:

H2: Social overload is negatively related to SNS satisfaction.

\subsection{Associations between Receiving Social Support and SNS Exhaustion and SNS Satisfaction}

In the context of this paper, receiving social support refers to the extent to which SNS users feel they are supported and being taken care of by others in their personal SNS network. This support can occur if users feel that their SNS friends care for them and their well-being on the SNS. Social support also transpires if users feel that their SNS friends take the time to deal with their problems. In the field of medicine, the effect of social support affords both physical and psychological benefits [e.g. $28,33,36]$ and the benefits can be both direct and indirect $[36,44]$. In this paper, we concentrate on the direct effects of social support. In the study mentioned earlier of older people suffering from arthritis, it was found that regardless of the severity of arthritic conditions, those who had the support of close social relationships had better psychological functioning and experienced less distress [36]. Likewise, in a separate study, injured athletes receiving social support felt less restless, isolated, and cheated [28]. Because receiving social support tends to reduce distress and other stress-related factors as well as improve psychological functioning, the applicability of considering social support in the context of SNS use properly applies here. Similar to the offline context in the medical studies, receiving social support on a SNS should also reduce stressrelated factors, in this case reduce a user's SNS exhaustion. Consequently,

\section{H3: Receiving social support is negatively related to SNS exhaustion.}

Not only should social support reduce SNSrelated exhaustion, but SNS users who receive social support from their SNS friends should also likely be more satisfied with the SNS. The buffer effect of social support theory suggests that receiving social support improves both physical and psychological well-being [44]. Studies have found that supportive online interactions enhance positive affect and indirectly, life satisfaction [e.g. 34]. This suggests that receiving social support on a SNS, which represents a supportive online interaction, leads to positive affective feelings, included of which are feelings of satisfaction. Found in previous studies is that social overload can reduce SNS satisfaction, and here the opposite can be true: receiving social support can improve SNS satisfaction. So it is hypothesized: 
H4: Receiving social support is positively related to SNS satisfaction.

\subsection{Associations among SNS Exhaustion, SNS Satisfaction, and Discontinuous Usage Intention}

The last three hypotheses have been suggested in prior research $[25,26]$. The first of these suggests that SNS exhaustion reduces SNS satisfaction. The relationship between feelings of exhaustion and feelings of satisfaction can be found in other contexts as well. For example, in studies of job turnover, it has been consistently found that work exhaustion leads to job dissatisfaction [40]. Employees who feel overwhelmed by work-related stressors are less satisfied with their jobs. Similarly, in the current context, users who feel exhausted using a SNS would also feel less satisfied with the SNS. We therefore hypothesize:

H5: SNS exhaustion is negatively related to SNS satisfaction.

The second and third of the last three hypotheses suggest that SNS exhaustion increases a user's discontinuous usage intention while SNS satisfaction reduces discontinuous usage intention. Discontinuous usage intention refers to a user's intention to reduce the intensity of their SNS usage or to completely stop using the SNS altogether [26]. In the work-related context, negative psychological consequences such as work exhaustion will increase an employee's intention to leave a job, whereas positive psychological consequences such as job satisfaction will reduce an employee's intention to leave the job [30]. The application of those relationship associations from the work-related context should correspond to the SNS context such that a user's exhaustion with a SNS should increase his or her discontinuous usage intention, whereas a user's satisfaction with the SNS should reduce his or her discontinuous usage intention. It is thus hypothesized:

H6: SNS exhaustion is positively related to discontinuous usage intention.

H7: SNS satisfaction is negatively related to discontinuous usage intention.

\subsection{Exploratory Research Question}

To address the second purpose of this paper, which was to explore the effects of receiving social support for people who are lonely or emotionally unstable, we propose the following exploratory research question.

Exploratory question: How do the results of the above research model differ for those users who are lonely or emotionally unstable?

By testing the research model and comparing the results for people who are lonely or emotionally unstable as opposed to those who are not, we begin to understand whether and how much social overload and receiving social support impact their psychological responses of SNS exhaustion and SNS satisfaction.

\section{Method}

We chose to examine Facebook as the context for this study. Since Facebook boasts the largest number of users of any SNS [4], we reasoned that the potential participant pool would be greatest for this site than any other SNS. An online questionnairebased study was conducted to examine the proposed research model. Announcements, primarily in the form of an email, were sent to two university communities to solicit participation for the study. One community is a medium to large university located in the eastern United States and the other is a small to medium sized university located in the Midwestern United States. Potential respondents included all staff, faculty, and students of adult age. The potential respondent pool is predominantly female: 54 to $59 \%$ of staff and faculty are female and 56 to $60 \%$ of students are female. To incentivize study participation, respondents were given an option to enter a raffle to win one of six $\$ 50$ gift cards to a store of their choosing. A total of 1363 responses were recorded. Data cleansing yielded 1285 usable responses for analysis. Because we had a sufficient number of responses, responses that had more than $15 \%$ missing data, reflecting early participant attrition, were cleansed from the sample, as recommended [17].

\subsection{Measures}

All measures for the study were either adopted or adapted from prior literature. Most constructs were measured using multi-item, 7-point response scales ranging from "strongly disagree" to "strongly agree", except loneliness, which was measured using a 5point scale ranging from "never" to "always", following extant literature. Construct items and their corresponding sources can be found in the Appendix. All variables were modeled as reflective constructs. 


\section{Analysis and Results}

After data cleansing, a total of 1285 usable responses were retained for further analysis. The demographic characteristics of the respondents are shown the Table 1.

\begin{tabular}{|l|l|l|}
\hline \multicolumn{3}{|c|}{ Table 1. Sample Characteristics } \\
\hline Attributes & Count & Percentage \\
\hline Age & 437 & $34 \%$ \\
\hline 18 to 20 & 455 & $35 \%$ \\
\hline $21-30$ & 138 & $11 \%$ \\
\hline $31-40$ & 102 & $8 \%$ \\
\hline $41-50$ & 153 & $12 \%$ \\
\hline $51+$ & \multicolumn{2}{|l|}{} \\
\hline Sex & 281 & $22 \%$ \\
\hline Male & 1004 & $78 \%$ \\
\hline Female & 114 & $9 \%$ \\
\hline Education & 551 & $43 \%$ \\
\hline High school & 95 & $7 \%$ \\
\hline Some college & 239 & $19 \%$ \\
\hline Associate's & 147 & $11 \%$ \\
\hline Bachelor's & 133 & $10 \%$ \\
\hline Master's & 5 & $0 \%$ \\
\hline Doctorate &
\end{tabular}

\subsection{Bias Checks and Control Variables}

We examined the data for potential response biases and acknowledge that the sample is composed primarily of female respondents. There are several reasons that could account for this, which will be further discussed in the limitations section.

Next, common method bias was examined using Harman's single-factor test [37] and a Lindell and Whitney [24] test. Harman's test found no substantial common method variance since no single factor accounted for most of the variance. The Lindell and Whitney [24] test using a theoretically
Finally, control variables were tested prior to analyzing the research model to determine whether any were significant predictors of the dependent variable, discontinuous usage intention. Variables tested include age, sex, and education. Only age was found to be a significant predictor such that the older the user, the lower his or her discontinuous usage intention.

\subsection{Measurement Model}

The partial least squares (PLS) approach was used to examine the model. PLS, originally developed for econometrics, has become a popular approach to analyzing data in the social sciences, business, and education fields [17]. PLS is estimated with regression-based methods and focuses on maximizing explained variance, that is, prediction of the constructs, and avoids solution inadmissibility and factor indeterminacy problems, making it more suitable for exploratory studies [17]. The model was analyzed using SmartPLS 3 due to its functionality set and ease-of-use.

Since all measures for the model are reflective, reliability/convergent validity and discriminant validity measurement validations were performed. Reliability/convergent validity were confirmed by Cronbach's Alpha, composite reliability (CR), and average variance extracted (AVE). Cronbach's Alpha indicators of approximately .70 or greater [17] and CR measures of .70 or greater [5] indicate acceptable internal consistency. AVEs of .50 or greater indicate acceptable reliability and convergent validity [5, 12]. Table 2 provides the Cronbach's Alphas, CRs, and AVEs for each factor.

Discriminant validity was assessed in two ways. First, as illustrated in Table 2, the square root of the AVE for each factor was higher than the correlations with other factors, indicating that each factor shares

\begin{tabular}{|l|c|c|c|r|r|r|r|r|r|r|}
\hline \multicolumn{8}{|c|}{ Table 2. Measurement Validations and Correlations } \\
\hline & $\begin{array}{l}\text { Cronbach's } \\
\text { Alpha }\end{array}$ & CR & AVE & \multicolumn{1}{|c|}{1} & 2 & 3 & 4 & 5 & 6 & 7 \\
\hline 1. Discontinuous Usage Intention & 0.825 & 0.885 & 0.659 & 0.812 & & & & & & \\
\hline 2. Emotional Stability & 0.690 & 0.805 & 0.685 & -0.083 & 0.828 & & & & & \\
\hline 3. Loneliness & 0.870 & 0.902 & 0.607 & 0.126 & -0.464 & 0.779 & & & & \\
\hline 4. Receiving Social Support & 0.820 & 0.878 & 0.644 & -0.115 & -0.098 & 0.047 & 0.802 & & & \\
\hline 5. SNS Exhaustion & 0.921 & 0.944 & 0.808 & 0.423 & -0.173 & 0.255 & 0.057 & 0.899 & & \\
\hline 6. SNS Satisfaction & 0.914 & 0.946 & 0.854 & -0.493 & 0.015 & -0.123 & 0.233 & -0.359 & 0.924 & \\
\hline 7. Social Overload & 0.871 & 0.911 & 0.720 & 0.057 & -0.151 & 0.191 & 0.589 & 0.221 & 0.092 & 0.848 \\
\hline Note: The diagonal shaded cells are the square roots of the average variance extracted (AVE) for each factor. \\
\hline
\end{tabular}

unrelated variable, called a "marker" variable (in our case, the participant's time spent working each day), found very low correlations between a participant's time spent working each day and the model's constructs (average correlation $r=0.018$ ), which indicates minimal evidence of common method bias. higher variance with items in its own factor than with items in other factors. Second, as shown in Table 3, the loadings and cross-loadings indicate that each item loads higher on its own construct than on other constructs. These assessments show discriminant validity [5]. In the process of achieving satisfactory 


\begin{tabular}{|c|c|c|c|c|c|c|c|}
\hline \multicolumn{8}{|c|}{ Table 3. Loadings and Cross Loadings } \\
\hline & $\begin{array}{l}\text { Discontinuous } \\
\text { Usage } \\
\text { Intention }\end{array}$ & $\begin{array}{l}\text { Emotional } \\
\text { Stability }\end{array}$ & Loneliness & $\begin{array}{l}\text { Receiving } \\
\text { Social } \\
\text { Support }\end{array}$ & $\begin{array}{l}\text { SNS } \\
\text { Exhaustion }\end{array}$ & $\begin{array}{l}\text { SNS } \\
\text { Satisfaction }\end{array}$ & $\begin{array}{l}\text { Social } \\
\text { Overload }\end{array}$ \\
\hline DiscontUseInt 1 & 0.756 & -0.025 & 0.074 & -0.082 & 0.369 & -0.425 & 0.066 \\
\hline DiscontUseInt_2 & 0.874 & -0.060 & 0.134 & -0.118 & 0.349 & -0.415 & 0.029 \\
\hline DiscontUseInt 3 & 0.879 & -0.086 & 0.122 & -0.081 & 0.355 & -0.424 & 0.055 \\
\hline DiscontUseInt 4 & 0.728 & -0.036 & 0.073 & -0.067 & 0.292 & -0.326 & 0.032 \\
\hline EmoStability_2 & -0.013 & 0.825 & -0.400 & -0.071 & -0.147 & 0.055 & -0.141 \\
\hline Loneliness 1 & 0.095 & -0.352 & 0.772 & 0.018 & 0.177 & -0.080 & 0.157 \\
\hline Loneliness_2 & 0.096 & -0.369 & 0.792 & 0.043 & 0.199 & -0.074 & 0.166 \\
\hline Loneliness 4 & 0.087 & -0.419 & 0.788 & 0.048 & 0.184 & -0.071 & 0.116 \\
\hline Loneliness 5 & 0.126 & -0.418 & 0.864 & 0.051 & 0.243 & -0.132 & 0.150 \\
\hline Loneliness_6R & 0.081 & -0.314 & 0.692 & 0.031 & 0.157 & -0.096 & 0.174 \\
\hline Loneliness_8 & 0.094 & -0.397 & 0.759 & 0.055 & 0.217 & -0.113 & 0.136 \\
\hline ReceivSupport_2 & 0.002 & -0.129 & 0.123 & 0.751 & 0.144 & 0.101 & 0.585 \\
\hline ReceivSupport 3 & -0.051 & -0.078 & 0.064 & 0.849 & 0.102 & 0.158 & 0.541 \\
\hline ReceivSupport 4 & -0.159 & -0.074 & -0.001 & 0.800 & -0.035 & 0.265 & 0.365 \\
\hline SNSExhaus_1 & 0.376 & -0.125 & 0.214 & 0.048 & 0.891 & -0.309 & 0.176 \\
\hline SNSExhaus 2 & 0.378 & -0.163 & 0.244 & 0.059 & 0.918 & -0.313 & 0.197 \\
\hline SNSExhaus_3 & 0.418 & -0.149 & 0.235 & 0.041 & 0.918 & -0.367 & 0.181 \\
\hline SNSExhaus 4 & 0.345 & -0.213 & 0.222 & 0.086 & 0.867 & -0.296 & 0.245 \\
\hline SNSSat_1 & -0.460 & 0.051 & -0.122 & 0.201 & -0.347 & 0.938 & 0.067 \\
\hline SNSSat 2 & -0.416 & 0.026 & -0.111 & 0.241 & -0.296 & 0.908 & 0.136 \\
\hline SNSSat_3 & -0.487 & 0.012 & -0.109 & 0.191 & -0.350 & 0.925 & 0.057 \\
\hline SocialOverload_1 & -0.008 & -0.093 & 0.116 & 0.522 & 0.107 & 0.127 & 0.782 \\
\hline SocialOverload 2 & 0.083 & -0.160 & 0.196 & 0.450 & 0.242 & 0.022 & 0.859 \\
\hline SocialOverload 3 & 0.063 & -0.144 & 0.177 & 0.517 & 0.201 & 0.081 & 0.873 \\
\hline SocialOverload 4 & 0.034 & -0.142 & 0.144 & 0.570 & 0.174 & 0.105 & 0.877 \\
\hline
\end{tabular}

reliability and validity, two items with loadings of less than 0.60 were dropped for loneliness, as noted in the Appendix.

\subsection{PLS Structural Model}

A final bootstrap resampling procedure of 5000 samples was used to test the significance of the structural research model. In the presence of the model factors, the control variable age accounted for only $1.8 \%$ of the difference in $\mathrm{R}^{2}$ and impact on the model was negligible and so was removed. Figure 2 illustrates the overall model results from the structural analysis, with explanatory powers $\left(\mathrm{R}^{2}\right)$ and standardized path coefficients $(\beta)$ as depicted. The results show that 6 of the 7 hypotheses were supported. SNS exhaustion and SNS satisfaction explain $31 \%$ of the variance for users' discontinuous usage intention. The receiving social support hypotheses were found to be significantly related to both SNS exhaustion and SNS satisfaction.

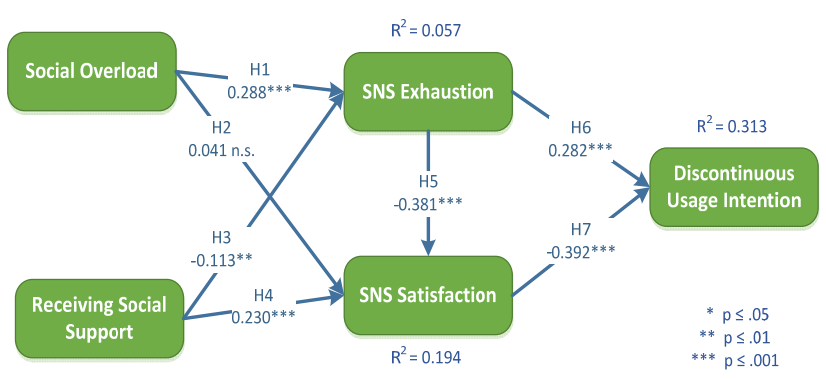

Figure 2. Overall Model Results

\subsection{Exploratory Results}

The second purpose of this paper was to explore the effects of the model for people who are lonely or emotionally unstable as opposed to people who are not. The question, "How do the results of the above research model differ for those users who are lonely or emotionally unstable?" was posed earlier. To address this, the sample was divided two different times: one time based on loneliness and another time based on emotional stability. Since responses for loneliness is based on a 5-point scale, we divided the sample such that responses above the midpoint 


\begin{tabular}{|c|c|c|c|c|c|c|c|}
\hline \multicolumn{8}{|c|}{ Table 4. Exploratory Results of Mean Comparisons } \\
\hline Construct & $\begin{array}{l}\text { Mean \& } \\
\text { Standard } \\
\text { Deviation }\end{array}$ & $\begin{array}{c}\text { Emotionally } \\
\text { Stable } \\
(\mathbf{n}=\mathbf{8 3 9})\end{array}$ & $\begin{array}{l}\text { Emotionally } \\
\text { Unstable } \\
(\mathbf{n}=285)\end{array}$ & $\begin{array}{c}\text { t-test Results } \\
\text { between High \& } \\
\text { Low Emotional } \\
\text { Stability Groups }\end{array}$ & $\begin{array}{c}\text { Not } \\
\text { Lonely } \\
(\mathbf{n}=978)\end{array}$ & $\begin{array}{c}\text { Lonely } \\
(n=307)\end{array}$ & $\begin{array}{l}\text { t-test Results } \\
\text { between Not Lonely } \\
\text { \& Lonely Groups }\end{array}$ \\
\hline \multirow{2}{*}{$\begin{array}{l}\text { Discontinuous } \\
\text { Usage } \\
\text { Intention }\end{array}$} & mean & 2.98 & 3.12 & \multirow{2}{*}{40} & 2.95 & 3.20 & \multirow[b]{2}{*}{0.004} \\
\hline & s.d. & 1.26 & 1.34 & & 1.22 & 1.38 & \\
\hline \multirow{2}{*}{$\begin{array}{l}\text { SNS } \\
\text { Satisfaction }\end{array}$} & mean & 4.52 & 4.44 & \multirow{2}{*}{ n.s. } & 4.55 & 4.35 & \multirow{2}{*}{0.012} \\
\hline & s.d. & 1.23 & 1.12 & & 1.19 & 1.23 & \\
\hline \multirow{2}{*}{$\begin{array}{l}\text { SNS } \\
\text { Exhaustion }\end{array}$} & mean & 2.72 & 3.18 & \multirow{2}{*}{0.000} & 2.73 & 3.25 & \multirow{2}{*}{0.000} \\
\hline & s.d. & 1.27 & 1.42 & & 1.29 & 1.40 & \\
\hline \multirow{2}{*}{$\begin{array}{l}\text { Social } \\
\text { Overload }\end{array}$} & mean & 2.22 & 2.48 & \multirow{2}{*}{0.001} & 2.23 & 2.54 & \multirow{2}{*}{0.000} \\
\hline & s.d. & 0.99 & 1.10 & & 0.98 & 1.12 & \\
\hline \multirow{2}{*}{$\begin{array}{l}\text { Receiving } \\
\text { Social Support }\end{array}$} & mean & 2.62 & 2.78 & \multirow{2}{*}{0.045} & 2.68 & 2.68 & \multirow{2}{*}{ n.s. } \\
\hline & s.d. & 1.15 & 1.19 & & 1.16 & 1.20 & \\
\hline
\end{tabular}

\begin{tabular}{|c|c|c|c|c|}
\hline \multicolumn{5}{|c|}{ Table 5. Exploratory Hypotheses Results } \\
\hline & $\begin{array}{c}\text { Emotionally } \\
\text { Stable }(n=839)\end{array}$ & $\begin{array}{c}\text { Emotionally } \\
\text { Unstable }(n=285)\end{array}$ & $\begin{array}{c}\text { Not Lonely } \\
(\mathrm{n}=978)\end{array}$ & $\begin{array}{c}\text { Lonely } \\
(\mathrm{n}=307)\end{array}$ \\
\hline \multicolumn{5}{|l|}{ Standardized Betas } \\
\hline H1: Social overload -> SNS exhaustion & $0.250^{* * *}$ & $0.303 * * *$ & $0.226 * * *$ & $0.382 * * *$ \\
\hline H2: Social overload -> SNS satisfaction & 0.005 n.s. & $0.161 *$ & 0.029 n.s. & 0.108 n.s. \\
\hline H3: Receiving social support -> SNS exhaustion & $-0.142 * * *$ & -0.155 n.s. & -0.057 n.s. & $-0.236^{*}$ \\
\hline H4: Receiving social support -> SNS satisfaction & $0.270 * * *$ & $0.154 *$ & $0.262 * * *$ & 0.114 n.s. \\
\hline H5: SNS exhaustion -> SNS satisfaction & $-0.360 * * *$ & $-0.462 * * *$ & $-0.371 * * *$ & $-0.402 * * *$ \\
\hline H6: SNS exhaustion -> Discontinuous usage intention & $0.237 * * *$ & $0.377 * * *$ & $0.239 * * *$ & $0.376^{* * *}$ \\
\hline H7: SNS satisfaction -> Discontinuous usage intention & $-0.419 * * *$ & $-0.379 * * *$ & $-0.396 * * *$ & $-0.385 * * *$ \\
\hline \multicolumn{5}{|l|}{ R-Squares } \\
\hline SNS Exhaustion & 0.044 & 0.059 & 0.039 & 0.086 \\
\hline SNS Satisfaction & 0.204 & 0.258 & 0.198 & 0.181 \\
\hline Discontinuous Usage Intention & 0.303 & 0.407 & 0.279 & 0.399 \\
\hline
\end{tabular}

Note: $* \mathrm{p} \leq .05 ; * * \mathrm{p} \leq .01 ; * * * \mathrm{p} \leq .001$

represent users who are lonely, responses below the midpoint represent users who are not lonely, and responses exactly at the midpoint are not included in the exploratory analysis because they represent neither. The two groups representing lonely users versus not lonely users were then analyzed. Starting with the entire sample again, the sample was then divided for responses based on emotional stability, similar to how it was done for loneliness, and analyzed. A comparison of the means for the model constructs can be found in Table 4. And the results of the structural model analyses for the groups can be found in Table 5.

It is interesting to point out that although prior studies have found the relationship between social overload and SNS satisfaction to be significantly negative [e.g. 25], our analysis does not confirm this either in the overall sample or in the different groups based on loneliness and emotional stability. More intriguing is the finding that for those who are emotionally unstable, this relationship actually becomes positive. More discussion of this and other study results are offered in the next section.

\section{Discussion}

In this paper, we first examined the effects of receiving social support and social overload in an overall model. Specifically, this model evaluates the extent to which these constructs affect SNS satisfaction, exhaustion, and indirectly, discontinuous usage intention. Second, we explored how these effects are likely to unfold for people who are lonely or emotionally unstable versus those who are not. By integrating findings from the field of medicine into studies of SNS exhaustion, we offer a framework that could pave the way for new research in post-adoption of social media for users who exhibit certain psychological characteristics.

Guided by the buffer effect of social support theory, this paper set out to introduce the concept of receiving social support as a deterrent of SNS exhaustion and a promoter of SNS satisfaction in the context of SNS discontinuous usage intention. In the overall model, the hypotheses regarding receiving social support were supported, confirming that the theory which emerged from the field of medicine has applicable and optimistic impacts in an online social networking context. However, the relationship between social overload and SNS satisfaction, which had been found in previous studies, is not confirmed. Overall, the results of this study do confirm extant findings regarding the relationships among SNS exhaustion, SNS satisfaction, and discontinuous usage intention. 
More thought stimulating are the exploratory research findings for those users who are emotionally unstable (i.e. neurotic) and lonely as opposed to those who are not. Prior studies that found social overload to be negatively associated with SNS satisfaction was not confirmed in the current study for the overall model nor for the individual subgroups, except for the group that is emotionally unstable (i.e. neurotic), but the finding for that group was opposite to what extent studies found. That is, instead of finding that greater social overload leads to lower SNS satisfaction, we found that greater social overload is associated with higher SNS satisfaction for neurotic users. This finding is rather difficult to explain, but a possible explanation might be that somehow neurotic users associate social overload with greater engagement and social activity which might temporarily distract them from their emotional issues and thereby increasing their SNS satisfaction. However, from literature, we observe that people who are emotionally unstable are more likely to feel more stressed in daily life and therefore feel less satisfaction with different aspects of their lives in general [20, 21]. For example, Hlatywayo et al. [20] found that bank employees who had lower levels of neuroticism also had higher levels of satisfaction with their jobs. And Judge and Bono [21] in a metaanalysis found that people who were more emotionally unstable had lower levels of job satisfaction. Additionally, neuroticism has been linked to an impaired ability to handle stress [29]. We were unable to find any studies suggesting that for people who are less emotionally stable, situational stress factors would increase situational satisfaction. Perhaps there are other factors in play that mediate or moderate this relationship and need to be further explored in future research.

Another interesting exploratory finding is that although emotionally unstable users receive significantly more social support on Facebook as compared to their emotionally stable counterparts, they are also significantly more socially overloaded and exhausted from their Facebook use (see Table 4). Perhaps because emotionally unstable people feel greater social overload, which leads to feelings of exhaustion, the effect of receiving social support on feelings of exhaustion is limited, thus the nonsignificant finding for exploratory H3 (see Table 5).

As for the SNS users who are lonely, receiving social support does seem to help reduce SNS exhaustion, but not improve SNS satisfaction. The lonely also experience significantly higher social overload, higher SNS exhaustion, less SNS satisfaction, and higher intentions to discontinue using Facebook than their non-lonely counterparts.
Perhaps as suggested in prior research, spending so much time on social media could have detrimental effects on people who are lonely. SNSs are not helpful for such people.

\subsection{Contributions}

The above findings allow this paper to advance information systems literature in a couple of important ways. First, we theorized and validated the buffer effect of social support theory by showing that receiving social support serves well as a predictor of both SNS satisfaction and exhaustion. Second, the proposed framework offers researchers a new theoretical lens for identifying and measuring a construct that enhances SNS satisfaction and, simultaneously, reduces exhaustion, while the dominating studies on SNS-related technostress do not provide coverage in this area $[25,27,42]$. Some of them focus on the antecedents of negative perceptions or feelings of SNS fatigue [49]. Others stress the formation and consequences of social overload [e.g., 26]. This paper, however, sheds light on an overlooked factor - the buffer effect of social support - that empirically shows its significant impacts on positive and negative antecedents of SNS discontinuous usage intention.

Some prior literature [e.g., 26] did treat SNS exhaustion and satisfaction as antecedents of discontinuous usage intention and our study validates the extant literature. Our results indicate that SNS exhaustion and SNS satisfaction explain $31 \%$ of the variance for users' discontinuous usage intention. Hence, this paper confirms a relationship between satisfaction/exhaustion and the dependent variable discontinuous usage intention.

However, some of our results do not agree with prior literature [e.g., 25] which indicated that the relationship between social overload and SNS satisfaction is significantly negative. Intriguingly, for individuals who experience emotional instability, our study reveals a positive relationship between social overload and satisfaction. Namely, greater social overload is associated with higher SNS satisfaction for emotionally unstable users. Regarding this finding, we posit that since emotionally unstable individuals are often associate with unusual use of SNS [42], their psychological response to social overload exhibits distinctive, unpredictable, or contrary patterns, compared to individuals who do not exhibit the same emotional irregularities. 


\subsection{Implications for Practice}

Practitioners from different sectors should recognize that using SNSs exerts impacts on individuals' behaviors as well as their psychological status. SNS providers have a high stake in users' continuous adoption to keep their key assets and remain financially sustainable [47]. Corporations (e.g., Toyota) that implement internal SNSs to strengthen communications and stimulate cooperation [48] also have a vested interest in reducing the negative consequences of social overload. Our study provides practitioners a way to mitigate the negative impacts of social overload by suggesting that perceptions of stress related to SNS use might be weakened by amplifying the buffer effect of social support. In terms of solutions, using peer-to-peer support (e.g., informal sub-circles within a social network) might reduce members' urge to leave a SNS. SNS providers might put internal networks in place to provide peer-to-peer support that fosters online relationships.

From the perspective of mental health practitioners, it might be prudent to suggest a reduction in people's time spent on social networks, especially for users who are prone to loneliness and neuroticism. The negatives of SNS use such as social overload and exhaustion seem to weigh more heavily on lonely and neurotic users as compared to other users, but without much counterbalance of the benefits associated with use such as greater satisfaction. The higher social support that neurotic users report receiving does not seem to outweigh the social overload they feel on the SNS. Lonely and neurotic users might reevaluate what the benefits of using SNSs are to them as opposed to the stresses associated with use and manage their SNS use accordingly.

On the other hand, given the benefits of receiving social support online, not all social media use is negative for lonely or neurotic users. Being part of a social media community can create a sense of belonging for those with emotional issues. Indeed, lonely people tend to be shy and lack social support and they turn to Facebook as a way to seek support [42]. These users, who commonly lack face-to-face social interactions, also go online to seek fulfillment of their need to affiliate [42]. If SNS providers can somehow offer features that would allow users to choose to immerse themselves in the positive aspects of SNS use (e.g. receiving social support) while minimizing the negative aspects (e.g. social overload), their sense of community and belonging can only grow. SNSs would serve as sources of encouragement rather than sources of stress, thereby effectively developing social media community.

\subsection{Limitations and Future Research}

One of the limitations of this study is that the sample is comprised of predominantly female respondents. Several reasons could account for this: 1) as previously mentioned, the potential respondent pool for the study leans toward female, 2) Facebook has more female users than male [45], 3) females are more active on Facebook than are males [45], and 4) in general, females are more likely to respond to surveys than are males $[9,31]$ and therefore our survey received more responses from females than males. A post-hoc t-test was performed on the five constructs in the research model as well as on emotional stability and loneliness to compare the mean differences between genders. No significant mean differences were found between genders, except for emotional stability and SNS satisfaction. Males had significantly higher emotional stability than females, while females were significantly more satisfied with the SNS than males. For the most part, having a predominantly female sample does not invalidate the generalizability of model results, but future research should examine a more balanced gender sample to confirm the current study findings.

Another limitation that can be addressed by future research is the lack of longitudinal data to confirm the results. A longitudinal study allows us to keep track of the buffer effect of social support over time. It is also beneficial to include a more diverse respondent sample to enhance the generalizability of research findings. In addition, we collected data only from users of Facebook. Despite the reasons why it is a valid context for the study, we cannot rule out that the context might have accounted for some of the differences in results from extant studies. Another contextual limitation might be that data was gathered from university settings and therefore may not represent users in general.

The finding that for emotionally unstable users, high social overload is associated with high SNS satisfaction suggests an area that future research should explore. Future research should identify the factors, either moderating or mediating, that explain the unique psychological functioning of emotionally unstable SNS users.

Finally, although this study aimed to test the relationship between receiving social support and SNS exhaustion/satisfaction, a more comprehensive framework is desired that would include other essential support variables that might offer buffering effects. 


\section{References}

[1] R. Alloway, and P. Bebbington, "The buffer theory of social support--a review of the literature", Psychological Medicine, (17:1), 1987, pp. 91-108.

[2] L.F. Bright, S.B. Kleiser, and S.L. Grau, "Too much Facebook? An exploratory examination of social media Fatigue", Computers in Human Behavior, (44), 2015, pp. 148-155.

[3] K.J. Cha, and E.M. Lee, "An empirical study of discontinuous use intention on SNS: From a perspective of society comparison theory", Journal of Society for eBusiness Studies, (20:3), 2015, pp. 59-77.

[4] D. Chaffey, "Global social media research summary 2016", available at http://www.smartinsights.com/socialmedia-marketing/social-media-strategy/new-global-socialmedia-research/, 2016.

[5] W.W. Chin, "The partial least squares approach for structural equation modeling", In Modern Methods for Business Research, Marcoulides, G. A. (ed.). Lawrence Erlbaum Associates: Mahwah, NJ, 1998, pp. 295-336.

[6] H.G. Chou, and N. Edge, "They are happier and having better lives than I am: The impact of using Facebook on perceptions of others' lives", Cyberpsychology, Behavior, and Social Networking, 2012, (15), pp. 117-121.

[7] S. Cohen, and H.M. Hoberman, "Positive events and social support as buffers on life change stress", Journal of Applied Social Psychology, (13:2), 1983, pp. 99-125.

[8] E. Crain, The digital detox that won't interrupt your life. Men's Journal, February 4. Accessed February 10, 2017 at http://www.mensjournal.com/expert-advice/the-digitaldetox-that-won-t-interrupt-your-life-20150204/bandevices-from-the-bedroom, 2015.

[9] R. Curtin, S. Presser, and E. Singer, "The effects of response rate changes on the index of consumer sentiment", Public Opinion Quarterly, (64), 2000, pp. 413-428.

[10] G.C.L. Davey, Social Media, Loneliness, and Anxiety in Young People, Psychology Today, December 15, https://www.psychologytoday.com/blog/why-weworry/201612/social-media-loneliness-and-anxiety-inyoung-people, 2016.

[11] J. Dick, Why Do Social Networks Increase Stress? Huffington Post, July 12, http://www.huffingtonpost.com/john-dick/social-networksand-stress b 3534170.html, 2013.

[12] C. Fornell, and D.F. Larcker, "Evaluating structural equation models with unobservable variables and measurement error", Journal of Marketing Research, (18:1), 1981, pp. 39-50.

[13] J. Fox, and J.J. Moreland, "The dark side of social networking sites: An exploration of the relational and psychological stressors associated with Facebook use and affordances", Computers in Human Behavior, (45), 2015, pp. 168-176.

[14] K.G. Giota, and G. Kleftaras, "The role of personality and depression in problematic use of social networking sites in Greece", Cyberpsychology: Journal of Psychosocial Research on Cyberspace, (7:3), 2013, http://dx.doi.org/10.5817/CP2013-3-6.

[15] S.D. Gosling, P.J. Rentfrow, and W.B. Swann Jr "A very brief measure of the Big-Five personality domains", Journal of Research in Personality, (37), 2003, pp. 504-528.

[16] N. Haferkamp, and N.C. Krämer, "Social comparison 2.0: Examining the effects of online profiles on socialnetworking sites", Cyberpsychology, Behavior, and Social Networking, (14), 2011, pp. 309-314.

[17] J.F. Hair, W.C. Black, B.J. Babin, and R.E. Anderson, Multivariate Data Analysis, 7th ed, 2009,Upper Saddle River, NJ: Prentice Hall.

[18] R.D. Hays, and M.R. DiMatteo, "A short-form measure of loneliness", Journal of Personality Assessment, (51:1), 1987, pp. 69-81.

[19] A. Heaps, The third metric: Managing techno-stress at work. Huffingtonpost.com, August 16. Accessed February $10, \quad 2017$ at http://www.huffingtonpost.com/allisonheaps/the-third-metric-managing-techno-stress-atwork b 5482695.html, 2014.

[20] C.K. Hlatywayo, T.S. Mhlanga, and T. Zingwe "Neuroticism as a determinant of job satisfaction among bank employees", Mediterranean Journal of Social Sciences, (4:13), 2013.

[21] T.A. Judge, and J.E. Bono, "Relationship of core selfevaluations traits-self-esteem, generalized self-efficacy, locus of control, and emotional stability-with job satisfaction and job performance: A meta-analysis", Journal of Applied Psychology, (86:1), 2001, pp. 80-92.

[22] E. Kross, P. Verduyn, E. Demiralp, J. Park, D.S. Lee, N. Lin, H. Shablack, J. Jonides, and O. Ybarra, "Facebook use predicts declines in subjective well-being in young adults", PLoS ONE, (8:8), 2013, e69841. doi:10.1371/journal.pone.0069841.

[23] C.C. Li, M.L. Chen, T.C. Chang, H.H. Chou, and M.Y. Chen, "Social support buffers the effect of selfesteem on quality of life of early-stage cervical cancer survivors in Taiwan", European Journal of Oncology Nursing, (19:5), 2015, pp. 486-494. 
[24] M.K. Lindell, and D.J. Whitney, "Accounting for common method variance in cross-sectional research designs", Journal of Applied Psychology, (86:1), 2001, pp. 114-121.

[25] C. Maier, S. Laumer, A. Eckhardt, and T. Weitzel, "When social networking turns to social overload: Explaining the stress, emotional exhaustion, and quitting behavior from social network sites' users", Proceedings of the European Conference on Information Systems, 2012, Barcelona, Spain.

[26] C. Maier, S. Laumer, A. Eckhardt, and T. Weitzel, "Giving too much social support: social overload on social networking sites", European Journal of Information Systems, (24:5), 2014, pp. 447-464.

[27] C. Maier, S. Laumer, C. Weinert, and T. Weitzel, "The effects of technostress and switching stress on discontinued use of social networking services: A study of Facebook use", Information Systems Journal, (25:3), 2015, pp. 275308 .

[28] I. Mitchell, L. Evans, T. Rees, and L. Hardy, "Stressors, social support, and tests of the buffering hypothesis: Effects on psychological responses of injured athletes", British Journal of Health Psychology, (19:3), 2014, pp. 486-508.

[29] C. Mohiyeddini, S. Bauer, and S. Semple, "Neuroticism and stress: The role of displacement behavior", Journal of Anxiety, Stress, \& Coping, (28:4), 2015, pp. 391-407.

[30] J.E. Moore, "One road to turnover: An examination of work exhaustion in technology professionals", MIS Quarterly, (24:1), 2000, pp. 141-168.

[31] D.L. Moore, and J. Tarnai, "Evaluating nonresponse error in mail surveys", In Survey Nonresponse, Groves, R. M., Dillman, D. A., Eltinge, J. L., and Little, R. J. A. (eds.). John Wiley \& Sons: New York, 2002, pp. 197-211.

[32] I. Pantic, "Online Social Networking and Mental Health", Cyberpsychology, Behavior, and Social Network, (17:10), 2014, pp. 652-657.

[33] K.B. Nucholls, J. Callell, and B.H. Kaplin, "Psycholsocial assets, life crisis and the prognosis of pregnancy", American Journal of Epidemiology, (95:5), 1972, pp. 431-41.

[34] H.J. Oh, E. Ozkayab, and R. LaRose, "How does online social networking enhance life satisfaction? The relationships among online supportive interaction, affect, perceived social support, sense of community, and life satisfaction", Computers in Human Behavior, (30:1), 2014, pp. 69-78.

[35] K.J. Park and S.B. Park "A study on the stress of using social networking services and its discontinuance intention", Journal of the Korea Society of Computer and Information, (19:12), 2014, pp. 275-285.

[36] B.W.J.H. Penninx, T. Van Tilburg, D.J.H. Deeg, D.M.W. Kriegsman, A.J.P. Boeke, and J.T.M. Van Eijk, "Direct and buffer effects of social support and personal coping resources in individuals with arthritis", Social Science \& Medicine, (44:3), 1997, pp. 393-402.

[37] P.M. Podsakoff, S.B. MacKenzie, J.Y. Lee, and N.P. Podsakoff, "Common method biases in behavioral research: A critical review of the literature and recommended remedies", Journal of Applied Psychology, (88:5), 2003, pp. 879-903.

[38] D. Roberts, Reboot or die trying. Outsideonline.com, September 2. Accessed February 10, 2017 at https://www.outsideonline.com/1926796/reboot-or-dietrying, 2014.

[39] D.W. Russell, "UCLA Loneliness Scale (Version 3): Reliability, validity, and factor structure", Journal of Personality Assessment (66:1), 1996, pp. 20-40.

[40] P.S. Rutner, B.C. Hardgrave, and D.H. McKnight "Emotional dissonance and the information technology professional”, MIS Quarterly, (32:3), 2008, pp. 635-652.

[41] P. Sandle, Many Brits seek digital 'detox' from average 25 hours per week online. Yahoo.com, August 3. Accessed August 4, 2016 at https://www.yahoo.com/news/manybrits-seek-digital-detox-average-25-hours230955373.html,2016.

[42] H. Song, A. Zmyslinski-Seelig, J. Kim, A. Drent, A. Victor, K. Omori, and M. Allen, "Does Facebook make you lonely?: A meta-analysis", Computers in Human Behavior, (36), 2014, pp. 446-452.

[43] P.A. Thoits, "Conceptual, methodological, and theoretical problems in studying social support as a buffer against life stress", Journal of Health and Social Behavior, (23:2), 1982, pp. 145-159.

[44] P.A. Thoits, Social support and psychological wellbeing: Theoretical possibilities. In Social Support: Theory, Research and Applications, Sarason, I. G., and Sarason, B. R. (eds.). Springer: Netherlands, 1985, pp. 51-72.

[45] I. Vermeren, Men vs. Women: Who Is More Active on Social Media? Brandwatch.com, January 28. Accessed January 6, 2017 at https://www.brandwatch.com/blog/menvs-women-active-social-media/, 2015.

[46] K. Wilson, S. Fornasier, and K.M. White, "Psychological predictors of young adults' use of social networking sites", Cyberpsychology, Behavior, and Social Networking, (13), 2010, pp. 173-177.

[47] T. Xu, Y. Yang, Z. Cheng, and J. Lim, "Retaining and attracting users in social networking services: an empirical 
investigation of cyber migration", The Journal of Strategic Information Systems, (23), 2014, pp. 239-253.

[48] M.H. Yokoyama, "How social network sites (SNS) have changed the employer-employee relationship and what are the next challenges for human resource (HR)", Human Resources and Organizations, (23:1), 2016, pp. 2-9.
[49] S. Zhang, L. Zhao, Y. Lu, and J. Yang, "Do you get tired of socializing? An empirical explanation of discontinuous usage behaviour in social network services", Information \& Management, (53:7), 2016, pp. 904-914.

\section{Appendix}

\section{Construct Items and Sources}

\section{Social Overload $^{\text {a }}$ (Source: Maier et al. 2014)}

1. I care too much for my friends' well-being on Facebook.

2. I deal too much with my friends' problems on Facebook.

3. My sense of being responsible for how much fun my friends have on Facebook is too strong.

4. I am too often caring for my friends on Facebook.

Receiving Social Support ${ }^{\text {a }}$ (Source: adapted based on Maier et al. 2014 social overload)

1. My Facebook friends take a lot of care about my well-being on Facebook.

2. My Facebook friends deal with a lot of my problems on Facebook.

3. My friends on Facebook take the care to ensure that I'm having fun on Facebook.

4. I get a lot of social support from my friends on Facebook.

SNS Exhaustion ${ }^{\text {a }}$ (Source: Maier et al. 2014)

1. I feel drained from activities that require me to use Facebook.

2. I feel tired from my Facebook activities.

3. Using Facebook is a strain for me.

4. I feel stressed out from my Facebook activities.

SNS Satisfaction ${ }^{a}$ (Source: Maier et al. 2014)

1. I am very pleased with Facebook.

2. I am delighted with Facebook.

3. Overall, I am very satisfied with Facebook.

Discontinuous Usage Intention ${ }^{\text {a }}$ (Source: Maier et al. 2014, 2015)

1. I will deregister/deactivate my Facebook account soon.

2. I prefer to use alternatives instead of continuing to use Facebook.

3 . In the future, I prefer to use alternatives to Facebook.

4. In the near future, I will use Facebook far less than I do today.

Emotional Stability ${ }^{\text {a }}$ (Source: Gosling et al. 2003, part of the 10 item Big 5 personality measure)

1. Anxious, easily upset (R)

2. Calm, emotionally stable

Loneliness $^{\mathrm{b}}$ (Sources: Hays and DiMatteo 1987; Russell 1996, from the UCLA Loneliness Scale)

1. How often do you feel that you lack companionship?

2. How often do you feel that there is no one you can turn to?

3. How often do you feel outgoing and friendly? (R) [dropped]

4. How often do you feel left out?

5. How often do you feel isolated from others?

6. How often do you feel you can find companionship when you want it? (R)

7. How often do you feel shy? [dropped]

8. How often do you feel that people are around you but not with you?

Scales: a: 7-point scale ranging from (1) strongly disagree to (7) strongly agree; b: 5-point scale ranging from (1) never to (5) always 